\title{
Digital tech and the pandemic
}

For our 2021 technology of the year, we explore the impact of the coronavirus pandemic on digital technology.

n January 2019 we launched our technology of the year series. Intended to highlight an area that has achieved a key breakthrough or reached an important moment of development, and reflecting the thoughts of the editorial team, we chose edge computing that year and $5 \mathrm{G}$ the next. This year, one event - the coronavirus pandemic - has overshadowed all discussion. And the technology that provides the greatest hope for 2021 - COVID-19 vaccines - is a biotech one. Our focus is, of course, on the development and implications of electronic technology. Thus, for our 2021 technology of the year we have taken a different approach and highlight the impact of the pandemic on digital technology.

In the first of a series of Comment articles on the topic, Manuel Cebrian of the Max Planck Institute for Human Development in Berlin discusses the past, present and future of digital contact tracing. The potential of this technology to slow the spread of a virus had been explored by Cebrian, together with Kate Farrahi and Rémi Emonet, around a decade ago (PLoS ONE 9, e95133; 2014) - to little fanfare within academia or beyond. The COVID-19 pandemic then thrust it into the spotlight. But, as Cebrian argues, whether it can be effective in the hard-to-model complexity of real-world social networks remains uncertain.

Another digital technology that could potentially be of value in the fight against the virus is wearable electronic devices. Such devices can continuously monitor physiological signals, and in a News \& Views article in this issue, Ceren Ates, Ali Yetisen, Firat Güder and Can Dincer of the University of Freiburg and Imperial College London examine recent research on the use of smartwatch data to detect asymptomatic and pre-symptomatic cases of COVID-19. They then go on to consider the emerging capabilities of wearable electronic devices and how they could be applied in the fight against future pandemics.

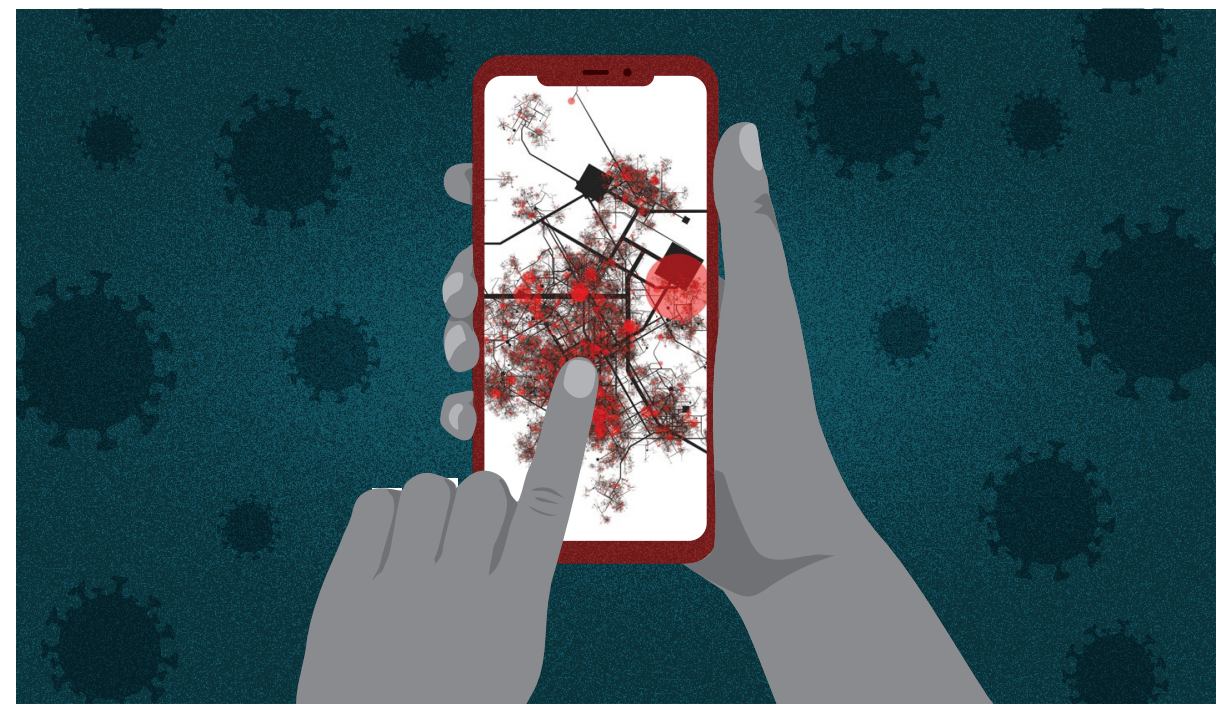

Credit: graphic in phone: Panther Media GmbH / Alamy Stock Photo

As well as highlighting the potential of emerging tech, the coronavirus pandemic has also had a disruptive effect on our relationship with everyday tech, creating shifts in approaches and expectations that may have long-term consequences. To start, the pandemic has forced students and teachers across all levels of education to quickly adapt to online learning. In a Comment article in this issue, Barbara Lockee of Virginia Tech examines the effects of this, the developments required to make it work, and whether the enforced change could have a permanent impact on how education is delivered.

As the virus has spread, people around the world have increasingly turned to social media for news and information. But the pandemic has been accompanied by a deluge of misleading and unreliable information. (This has included an unlikely and absurd connection to our 2020 technology of the year: a conspiracy theory that linked the spread of the coronavirus to $5 \mathrm{G}$ technology.) In response, social media platforms have taken steps to moderate content and promote official sources of information - actions that they had long been reluctant to undertake. In a further Comment article, Paul Butcher of the European Policy Centre in Brussels considers whether this could mark a turning point in the fight against both accidental misinformation and deliberate disinformation.

This increasing reliance on digital tech has also been accompanied by concerning trends in privacy and digital ethics, which again could have lasting effects. In our final Comment article, Carissa Véliz of the University of Oxford explores the issues surrounding privacy and digital ethics during the pandemic, and whether more robust protection of our rights in the digital realm is possible in the future.

Published online: 25 January 2021 https://doi.org/10.1038/s41928-021-00539-3 